\title{
In situ GIWAXS and GISAXS studies of surfactant-templated metal oxide film formation and thermal transformation
}

\author{
Stephen E. Rankina , M. Arif Khana, Yuxin He ${ }^{a}$, Syed Z. Islamª, Suraj Nagpure ${ }^{a}$, \\ Saikat Das ${ }^{a}$, Barbara L. Knutson ${ }^{a}$, and Joseph Strzalkab \\ a Department of Chemical and Materials Engineering, University of Kentucky, Lexington, KY \\ 40506-0046, USA, stephen.rankin@uky.edu \\ ${ }^{b}$ X-Ray Science Division, Argonne National Laboratory, Argonne, Illinois 60439, USA, \\ strzalka@anl.gov
}

The evaporation-induced self assembly (EISA) method was developed by the Brinker group [1] as a route to forming well-defined mesoporous thin films and particles. It uses evaporation of organic solvents to drive the co-assembly of metal oxide precursors with structure directing agents such as surfactants. Since its discovery, there have been many advances in understanding the mechanisms of film formation by dip coating, generation of ordered mesophases during aging, and transformation during heating, but there is still much to learn using advanced characterization techniques such as X-ray scattering.

Our group has made extensive use of grazing incidence wide- and small- angle X-ray scattering (GIWAXS and GISAXS) to understand how to control mesophase orientation and structure in silica and titania films templated using Pluronic surfactants. Our group has used these methods to follow the development of $\mathrm{TiO}_{2}$ films with vertically oriented hexagonal close packed pore channels due to surface chemistry modifications.[2] We also have used GIWAXS to show that the vertically oriented pores are stabilized against loss of mesostructure during crystallization of $\mathrm{TiO}_{2}$ during heat treatment.[3,4] Here I will describe recent efforts to investigate epitaxial growth of oriented multilayered F127-templated titania films (Fig. 1) and their transformation to films with vertically oriented mesopores. A similar mechanism is found in $\mathrm{TiO}_{2}$-doped $\mathrm{SiO}_{2}$ films, giving rise to small $(3 \mathrm{~nm})$ vertically aligned mesopores by using CTAB as a pore template.
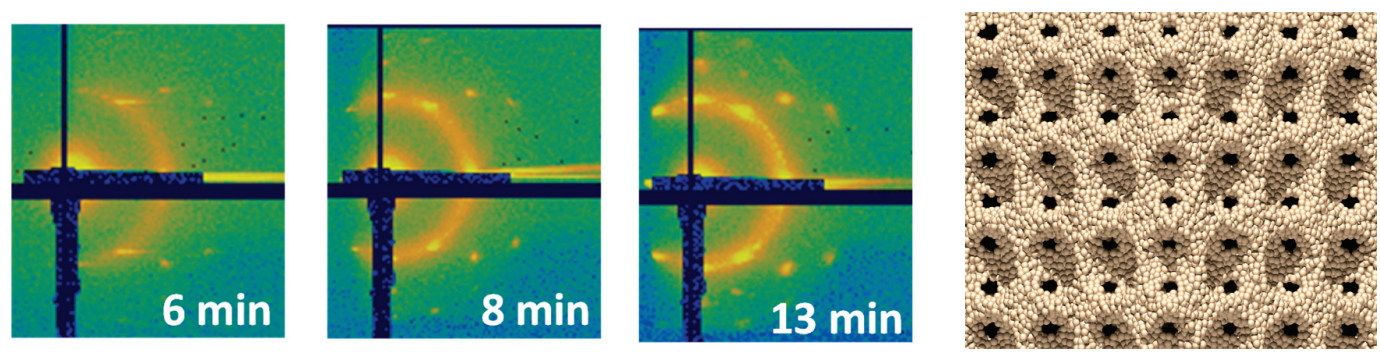

Fig. 1. Example of in situ GISAXS from a vertically aligned $\mathrm{TiO}_{2}$ precursor / Pluronic $\mathrm{F} 127$ dip coated solution. Times elapsed after dipping the sol onto vertically oriented glass slides at $4{ }^{\circ} \mathrm{C}$ and $78 \%$ relative humidity are indicated. The beam is reflected to the right of the film, giving rise to a set of diffraction spots indexed to the $\operatorname{Im} \overline{3} \mathrm{~m}$ (body-centered cubic) mesophase, a simulation of which is shown at right.

\section{References}

[1] Brinker, C.J., Y. Lu, A. Sellinger \& H. Fan (1999) Adv. Mater. 11, 579-585.

[2] Nagpure, S., S. Das, R.K. Garlapalli, J. Strzalka \& S.E. Rankin (2015) J. Phys. Chem. C 119, 2297022984.

[3] Das, S., Q. Wu, R.K. Garlapalli, S. Nagpure, J. Strzalka, Z. Jiang \& S.E. Rankin (2014) J. Phys. Chem. C 118, 968-976.

[4] Das, S., S. Nagpure, R.K Garlapalli, Q. Wu, S.Z Islam, J. Strzalka \& S.E. Rankin (2016) Phys. Chem. Chem. Phys. 18, 2896-2905. 